\title{
TRAVELING WAVE SOLUTIONS OF TWO-DIMENSIONAL NONLINEAR SCHRODINGER EQUATION VIA SINE-COSINE METHOD
}

\author{
Shaikhova G.N., Kutum B.B.
}

\author{
L.N. Gumilyov Eurasian National University, Nur-Sultan, Kazakhstan, kuttykadam@mail.ru
}

\begin{abstract}
In this work, an analytical study of the two-dimensional nonlinear Schrodinger equation is presented, namely, the applicability of the sine-cosine method to search for the exact solution as a traveling wave. The widely known nonlinear Schrödinger equation plays an important role in the study of the theory of nonlinear waves in various fields of physics and has a huge number of exact solutions. This equation describes the evolution of the changing amplitude of nonlinear waves in various systems, such as weakly nonlinear and highly dispersive. One of the methods for obtaining exact solutions is the sine-cosine method. The advantage of this method is its simplicity and reliability in obtaining solutions to nonlinear problems. According to the method, the nonlinear evolution equation is reduced to the associated ordinary differential equations by wave transformation and then solved by sine or cosine functions. As a result of the applicability of the sine-cosine method, the traveling wave solutions are obtained for a two-dimensional nonlinear Schrodinger equation. 2D-graphs and 3D-graphs of the obtained solutions are shown.
\end{abstract}

Keywords: two-dimensional, nonlinear Schrodinger equation, sine-cosine method, traveling wave, solution.

\section{Introduction}

Nonlinear equations with dissipation and dispersion effects that arise in scientific applications have been under the huge size of investigations. They have various applications in various fields of science, such as mathematical and chemical physics, solid state physics. Many vigorous methods, such as Hirota method [1-3], the extended tanh method [4-6], the sine-cosine method [6-8], Darboux transformation [9-13], Kudryashov method [14] and others were successfully applied to study these types of equations. One striking example of this type of equation is the nonlinear Schrödinger equation, which plays an important role in the theory of nonlinear waves.

The two-dimensional nonlinear Schrodinger equation

$$
\begin{aligned}
& i q_{t}+q_{x y}-v q=0, \\
& v_{x}+2\left(|q|^{2}\right)_{y}=0
\end{aligned}
$$

is a typical soliton equation with rich physical and mathematical applications [15-19]. This equation was proposed in [15] and was later deduced in [16], [17]. The conservation laws of equation (1)-(2) is studied in [18], rogue wave solutions are obtained by Hirota method in [19].

In this work we study the system of equations (1)-(2) by the sine-cosine method that have been extensively studied and widely applied for a wide variety of nonlinear problems [6-8].

\section{The scheme of sine-cosine method}

In this section, we describe the sine-cosine method [6]. According to the sine-cosine method by using a wave variable

$$
u(x, t)=u(x-c t)
$$

the partial differential equation 


$$
E_{1}\left(u_{t}, u_{x}, u_{x x}, u_{x x x}, \ldots\right)=0 \text {, }
$$

can be converted to ordinary differential equation

$$
E_{2}\left(u, u^{\prime}, u^{\prime \prime}, u^{\prime \prime \prime}, \ldots\right)=0 \text {. }
$$

Then the equation (5) is integrated as long as all terms contain derivatives where integration constants are considered zeros. The solutions of ordinary differential equation (5) can be expressed in the form

$$
u(x, t)=\lambda \cos ^{\beta}(\mu \xi), \quad|\xi| \leq \frac{\pi}{2 \mu},
$$

or

$$
u(x, t)=\lambda \sin ^{\beta}(\mu \xi),|\xi| \leq \frac{\pi}{\mu^{\prime}}
$$

where the parameters $\lambda, \mu$ and $\beta$ will be determined, and $\mu$ is wave number and $c$ is wave speed respectively [6]. The derivatives of (6) become

$$
\begin{aligned}
& \left(u^{n}\right)^{\prime}=-n \beta \mu \lambda^{n} \cos ^{n \beta-1}(\mu \xi) \sin (\mu \xi), \\
& \left(u^{n}\right)^{\prime \prime}=-n^{2} \mu^{2} \beta^{2} \lambda^{n} \cos ^{n \beta}(\mu \xi)+n \mu^{2} \lambda^{n} \beta(n \beta-1) \cos ^{n \beta-2}(\mu \xi),
\end{aligned}
$$

And the derivatives of (7) have next forms

$$
\begin{aligned}
& \left(u^{n}\right)^{\prime}=-n \beta \mu \lambda^{n} \sin ^{n \beta-1}(\mu \xi) \cos (\mu \xi), \\
& \left(u^{n}\right)^{\prime \prime}=-n^{2} \mu^{2} \beta^{2} \lambda^{n} \sin ^{n \beta}(\mu \xi)+n \mu^{2} \lambda^{n} \beta(n \beta-1) \sin ^{n \beta-2}(\mu \xi),
\end{aligned}
$$

and so on for the other derivatives. Applying (6)-(11) into the reduced ordinary differential equation (5) we obtain a trigonometric equation of $\cos ^{\beta}(\mu \xi)$ or $\sin ^{\beta}(\mu \xi)$ terms. Then, we determine the parameters by first balancing the exponents of each pair of cosine or sine to determine $\beta$. Next, we collect all coefficients of the same power in $\cos ^{k}(\mu \xi)$ or $\sin ^{k}(\mu \xi)$, where these coefficients have to vanish. The system of algebraic equations among the unknown $\beta$, $\lambda$, and $\mu$ will be given and from that, we can determine coefficients.

\section{Using the sine-cosine method}

We consider the two-dimensional nonlinear Schrodinger equation (1)-(2). By transformation

$$
q(x, y, t)=e^{i(a x+b y+d t)} Q(x, y, t),
$$

the equation (1)-(2) can be converted to

$$
\begin{aligned}
& i\left(i d Q+Q_{t}\right)+\left(-b a Q+i b Q_{x}+i a Q_{y}+Q_{x y}\right)-v Q=0, \\
& v_{x}+2\left((Q)^{2}\right)_{y}=0 .
\end{aligned}
$$

We separate real and imaginary part in the equation (13)-(14) and obtain

$-d Q-b a Q+Q_{x y}-v Q=0$,

$i Q_{t}+b Q_{x}+a Q_{y}=0$,

$v_{x}+2\left(Q^{2}\right)_{y}=0$

Substituting the wave transformation

$Q(x, y, t)=Q(\xi)=Q(x+y-c t)$,

$v(x, y, t)=V(\xi)=Q(x+y-c t)$,

into system of equation (15)-(17) we obtain that

$Q(-d-b a)+Q^{\prime \prime}-v Q=0$,

$Q^{\prime}(-c+b+a)=0$
$V^{\prime}+2\left(Q^{2}\right)^{\prime}=0$

From equation (21) we obtain that $c=b+a$. Than we integrate equation (22) and obtain

$V=-2 Q^{2}$.

Substituting (23) into (20) we get 
$Q(-d-b a)+Q^{\prime \prime}+2 Q^{3}=0$.

We solve the equation (24) by the sine-cosine method. According to method the solution of the (24) can be found by transformation

$Q=\lambda \cos ^{\beta}(\mu \xi)$,

and

$Q=\lambda \sin ^{\beta}(\mu \xi)$.

To find cosine solution we use (25) and its derivative

$Q^{\prime \prime}=-\mu^{2} \beta^{2} \lambda \cos ^{\beta}(\mu \xi)+\mu^{2} \lambda \beta(\beta-1) \cos ^{\beta-2}(\mu \xi)$.

Substitute (25) and (27) into (24)we get

$\lambda(-d-b a) \cos ^{\beta}(\mu \xi)-\mu^{2} \beta^{2} \lambda \cos ^{\beta}(\mu \xi)+\mu^{2} \lambda \beta(\beta-1) \cos ^{\beta-2}(\mu \xi)+2 \lambda^{3} \cos ^{3 \beta}(\mu \xi)=0$.

From (28) we find $\beta$ :

$\beta-2=3 \beta$ then $\beta=-1$.

Substitute (29) in (28) we obtain next equation

$\lambda(-d-b a) \cos ^{-1}(\mu \xi)-\mu^{2} \lambda \cos ^{-1}(\mu \xi)+2 \mu^{2} \lambda \cos ^{-3}(\mu \xi)+2 \lambda^{3} \cos ^{-3}(\mu \xi)=0$.

From the equation (30) we have the next sytem

$\cos ^{-1}(\mu \xi): \lambda(-d-b a)-\mu^{2} \lambda=0$,

$\cos ^{-3}(\mu \xi): 2 \mu^{2} \lambda+2 \lambda^{3}=0$.

From (31) we obtain

$\mu=i \sqrt{d+b a}$,

and from (32) we get

$\lambda=\sqrt{d+b a}$.

Substituting (33)-(34) into (25) and then obtained expression into (12) and (19) we have cosine solution

$$
\begin{aligned}
& q_{1}(\mathrm{x}, \mathrm{y}, \mathrm{t})=e^{i(a x+b y+d t)} \sqrt{d+b a} \times \cos ^{-1}(i \sqrt{d+b a} \times(x+y-c t)), \\
& v_{1}(x, y, t)=-2(d+b a) \times \cos ^{-2}(i \sqrt{d+b a} \times(x+y-c t)),
\end{aligned}
$$

where $c=b+a$.

By same way we can find sine solution

$$
\begin{aligned}
& q_{2}(\mathrm{x}, \mathrm{y}, \mathrm{t})=e^{i(a x+b y+d t)} \sqrt{d+b a} \times \sin ^{-1}(i \sqrt{d+b a} \times(x+y-c t)), \\
& v_{2}(x, y, t)=-2(d+b a) \times \sin ^{-2}(i \sqrt{d+b a} \times(x+y-c t)),
\end{aligned}
$$

where $c=b+a$.

2D-graphs and 3D-graphs formsof the obtained solutions are presented in next Fig.1-6.
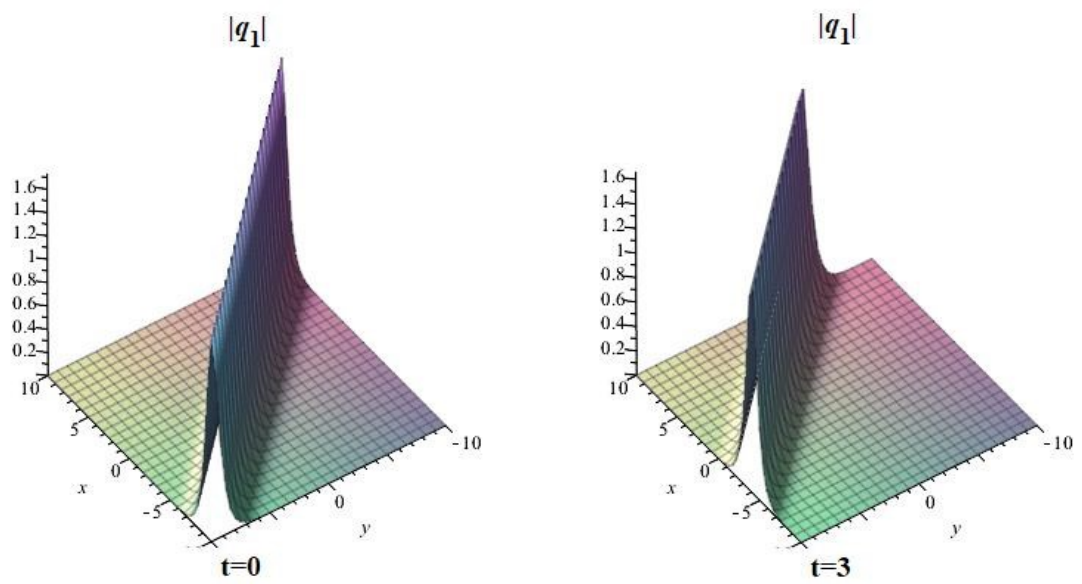

Fig.1. The 3D-graphs of the solution $q_{1}$ when $a=1, b=1, d=2$ 

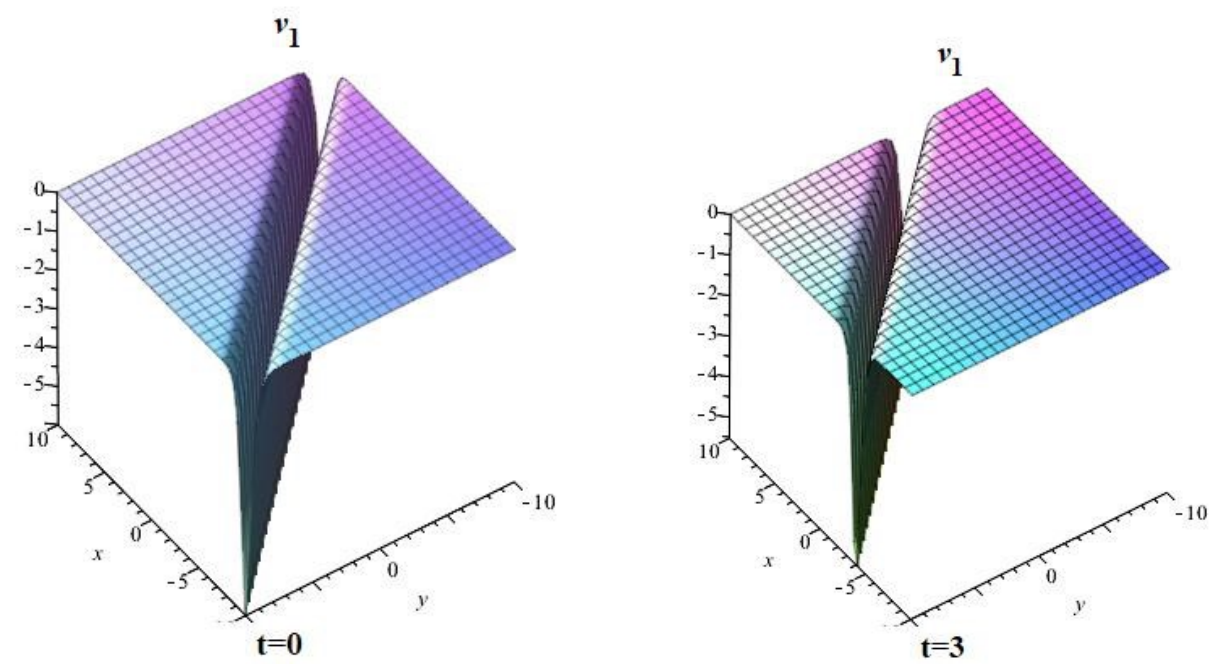

Fig. 2. The 3D-graphs of the solution $v_{1}$ when $a=1, b=1, d=2$
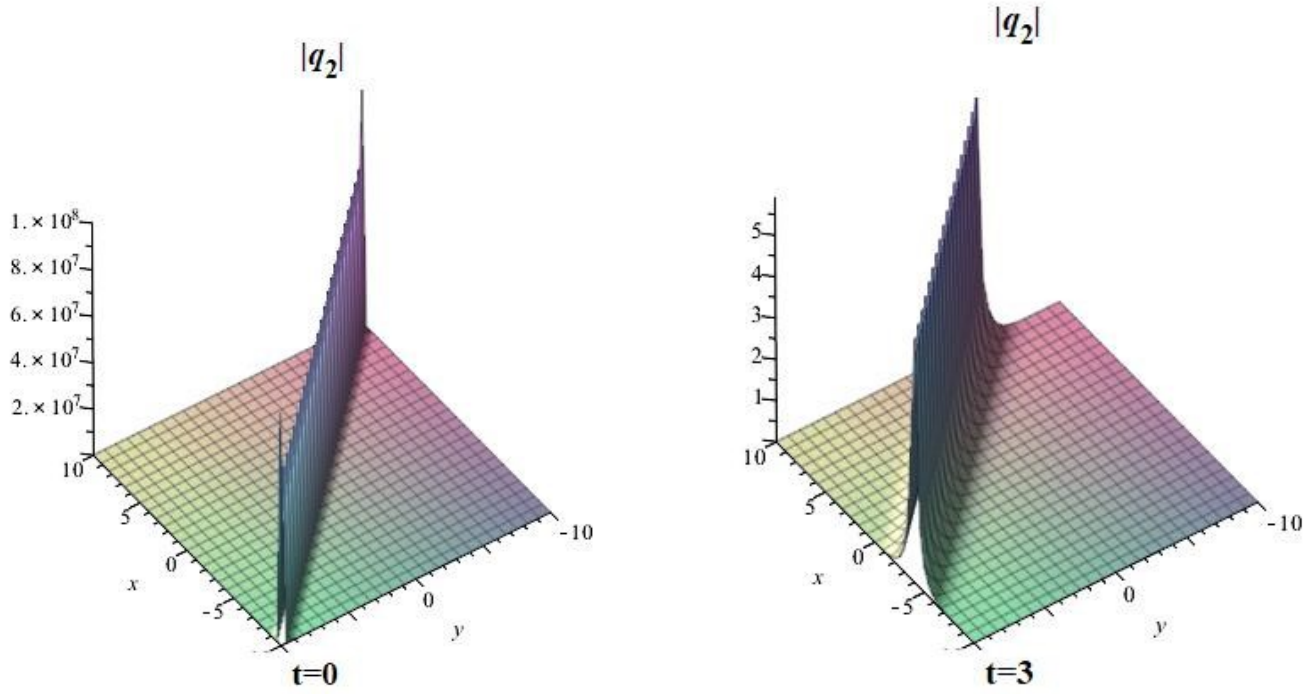

Fig.3. The 3D-graphs of the solution $q_{2}$ when $a=1, b=1, d=2$
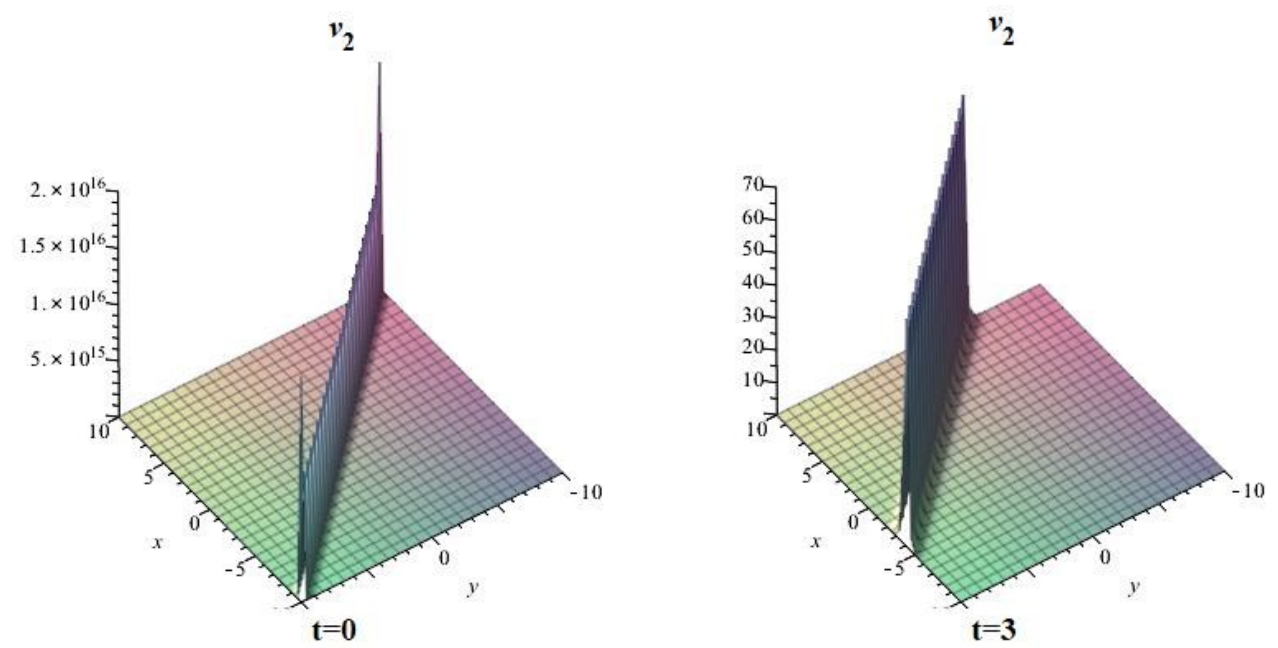

Fig. 4. The 3D-graphs of the solution $v_{2}$ when $a=1, b=1, d=2$ 

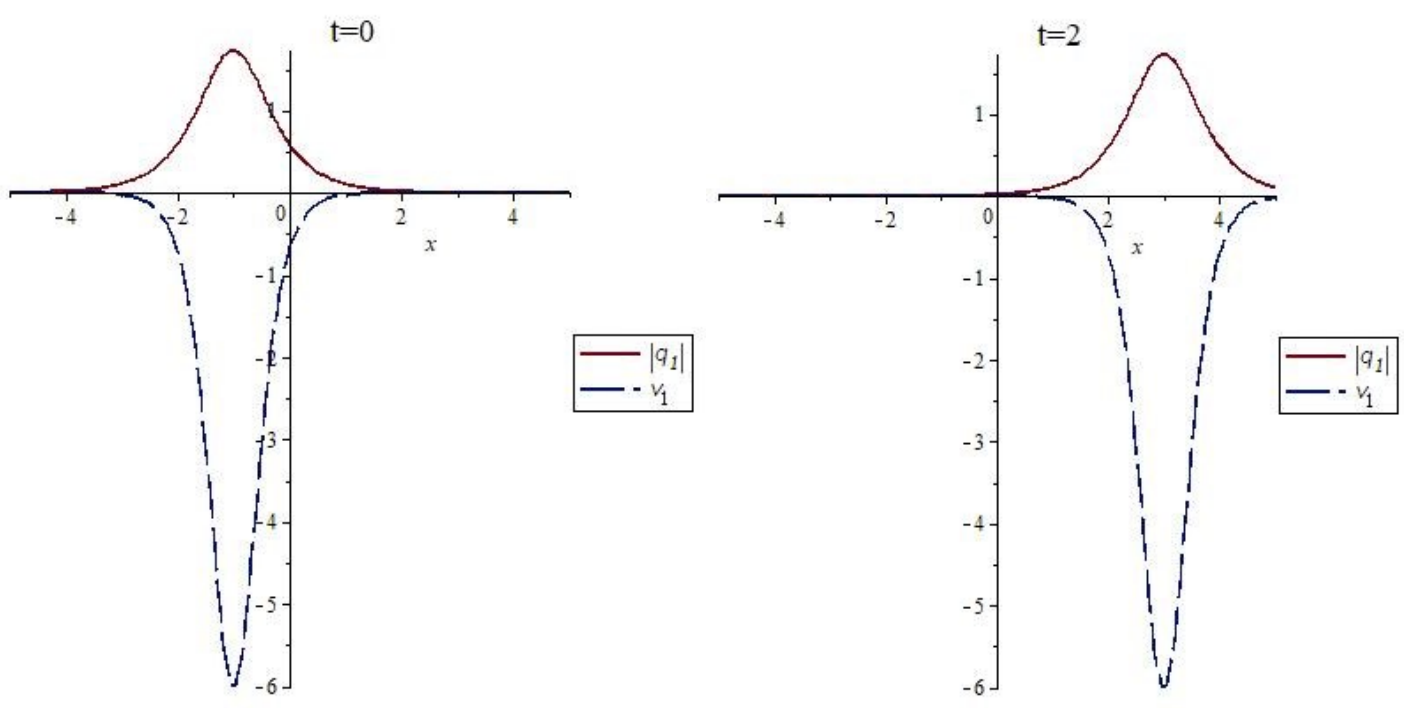

Fig. 5. The 2D-graphs of the solutions $q_{1}$ (solid line), $v_{1}$ (longdash line) with the parameters $a=1, b=1, d=2$
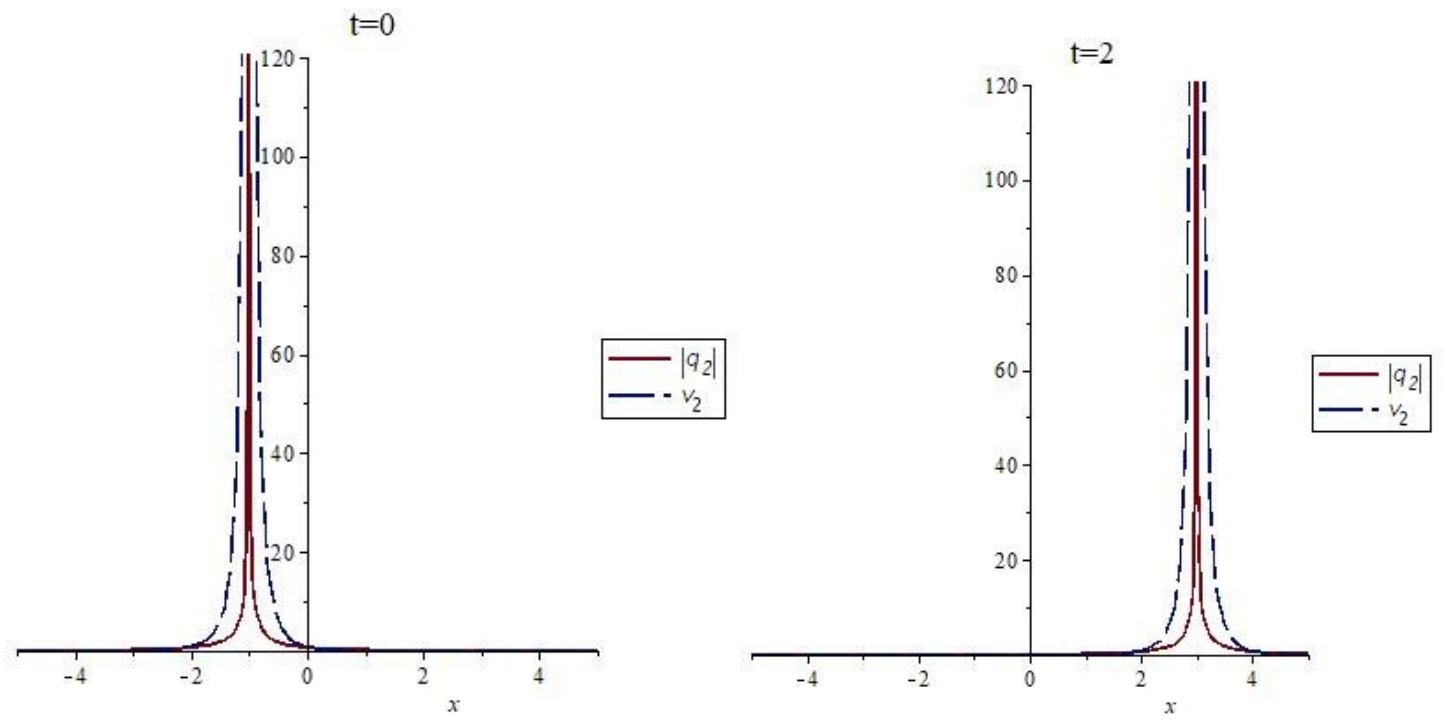

Fig. 6. The 2D-graphs of the solutions $q_{2}$ (solid line), $v_{2}$ (longdash line) with the parameters $a=1, b=1, d=2$

In Fig. 1 - 4 we present 3D plots of the travelling wave solutions (35)-(38). It can be seen that the waves keep their directions, widths, and amplitudes invariant during the propagation on the $x-$ $y$ plane.

Fig.5 displays the dynamics of the traveling wave in 2D plot. As we see the amplitude of solution $v_{1}$ (long dash line) bigger than the amplitude of solution $q_{1}$ (solid line).In Fig.6, we show the $2 \mathrm{D}$-graphs for the solutions $q_{2}$ (solid line) and $v_{2}$ (long dash line) with the parameters $\mathrm{t}=0$ and $\mathrm{t}=2$. As we notice solutions (37)-(38) are traveling to the right by saving shape.

\section{Conclusion}

In this work, we investigated the two-dimensional nonlinear Schrodinger equation by applying the sine-cosine method. The nonlinear Schrodinger equation has many applications in different areas of physics. We constructed various exact traveling wave solutions for this equation. The 
dynamics of the obtained traveling waves are shown in 2D-graphs and 3D-graphs forms. In figures, the traveling waves keep their directions, widths, and amplitudes invariant during the propagation. The sine-cosine method is a good mathematical tool for obtaining exact solutions for nonlinear wave equations in mathematical physics and other fields.

\section{Acknowledgements}

The article is performed as part of the financial support of the scientific and technical program (F. 0811, No. 0118RK00935) of the MES of the Republic of Kazakhstan.

\section{REFERENCES}

1 Mukhanmedina K.T., Syzdykova A.M., Shaikhova G.N. Soliton solutions of two-component Hirota equation. Bulletin of the Karaganda university. Mathematics series, 2015, No.4(80), pp. 103-107.

2 Kutum B.B., Shaikhova G.N. q-soliton solution for two-dimensional q-Toda lattice. Bulletin of the Karaganda University. Physics series, 2019, No.2(95), pp. 22-26.

3 Kutum B.B., Yesmakhanova K.R., Shaikhova G.N. The differential-q-difference 2D Toda equation: bilinear form and soliton solutions. Journal of Physics: Conference Series, 2019, Vol. 1391, 012122.

4 Yesmakhanova K., Bekova G., Shaikhova G. Travelling wave solutions for the two-dimensional Hirota system of equations.AIP Conf. Proc., 2018, Vol.1997, pp. 020039. doi:10.1063/1.5049033

5 Serikbayev N.S., Shaikhova G.N., Yesmakhanova K.R., Myrzakulov R. Traveling wave solutions for the (3+1)-dimensional Davey-Stewartson equations. Journal of Physics: Conference Series, 2019, Vol. 1391, pp. 012166.

6 Wazwaz A. Partial differential equations and solitary waves theory. 2009, Springer, $746 \mathrm{p}$.

7 Wazwaz A.M. A sine-cosine method for handling nonlinear wave equations. Mathematical and Computer Modeling, 2004, No 40(5), pp. 499-508.

8 Shaikhova G.N., Kutum B.B., Altaybaeva A.B., Rakhimzhanov B.K. Exact solutions for the $(3+1)-$ dimensional Kudryashov-Sinelshchikov equation. Journal of Physics: Conference Series, 2019, Vol.1416, pp. 012030.

9 Bekova G., Yesmakhanova K., Ozat N., Shaikhova G. Dark and bright solitons for the twodimensional complex modified Korteweg-de Vries and Maxwell-Bloch system with time-dependent coefficient. Journal of Physics: Conference Series, 2018,Vol. 965, pp.012035.

10 Yesmakhanova K., Shaikhova G., Bekova G., Myrzakulov R. Exact solutions for the $(2+1)-$ dimensional Hirota-Maxwell-Bloch system.AIP Conf. Proc., 2017,Vol. 1880, pp. 060022.

11 Yesmakhanova K., Bekova G., Myrzakulov R., Shaikhova G. Lax representation and soliton solutions for the (2+1)-dimensional two-component complex modified Korteweg-de Vries equations. Journal of Physics: Conference Series, 2017, Vol. 804, pp.012004.

12 Bekova G., Yesmakhanova K., Myrzakulov R, Shaikhova G. Darboux transformation and soliton solution for the (2+1)-dimensional complex modifed Korteweg-de Vries equations. Journal of Physics: Conference Series, 2017, Vol. 936, pp. 012045.

13 Bekova G., Shaikhova G., Yesmakhanova K., et al. Darboux transformation and soliton solution for generalized Konno-Ohno equation. Journal of Physics: Conference Series, 2019, Vol. 1416, pp. 012003.

14 Shaikhova G. Traveling wave solutions for the two-dimensional Zakharov-Kuznetsov-Burgers equation. Bulletin of the Karaganda University. Mathematics series, 2018, No.4(92), pp. 94-98.

15 Zakharov V.E. Solitons. Topics in Current Physics, 1980, Berlin, Springer, 389 p.

16 Strachan I.A.B. A new family of integrable models in $(2+1)$ dimensions associated with Hermitian symmetric spaces. J. Math. Phys., 1992, Vol. 33(7), pp. 2477-2482.

17 Strachan I.A.B. Some integrable hierarchies in $(2+1)$ dimensions and their twistor description. $J$. Math. Phys., 1993, Vol. 34(1), pp. 243-259.

18 Bekova G.T., Shaikhova G.N., Yesmakhanova K.R., Myrzakulov R. Conservation laws for two dimensional nonlinear Schrödinger equation. AIP Conference Proceedings, 2019, Vol. 2159, pp. 030003.

19 Rao J., Wang L., Liu W., He J. Rogue-wave solutions of the Zakharov equation. Theoretical and Mathematical Physics, 2017, Vol. 193(3), pp. 1783-1800. 